\title{
Evaluation of biomechanical loads for three-dimensional soaking postures in bathwater
}

\author{
Motomu NAKASHIMA*, Satoshi FUKUDA*, Takanao FUJII**, Rie SUZUKI**, Mamiko AZUMA** and \\ Ryota NAKAMURA** \\ *School of Engineering, Tokyo Institute of Technology \\ 2-12-2 Ookayama, Meguro-ku, Tokyo 152-8552, Japan \\ E-mail: motomu@sc.e.titech.ac.jp \\ ${ }^{* *}$ Research Institute, TOTO LTD \\ 2-8-1Honson, Chigasaki-city, Kanagawa 253-8577, Japan
}

Received: 6 February 2020; Revised: 29 June 2020; Accepted: 8 September 2020

\begin{abstract}
Comfort is required for soaking in bathwater. Although several studies investigated comfort during soaking from a biomechanical viewpoint, these previous studies employed two-dimensional models in the sagittal plane of a bather. The objectives of this study were to take three-dimensional postures of the bather into account and to evaluate the biomechanical loads for three-dimensional soaking postures. A three-dimensional biomechanical model was constructed, and an experiment to measure the soaking postures and reaction forces from the bathtub was conducted for eight participants. In addition, a supplementary experiment to measure the passive elastic joint torques was conducted for the same participants as well. Using the experimental results, the joint torques during soaking were calculated. In addition, the biomechanical load during soaking was defined as the sum of weighted joint torques, and the weighting coefficients were determined so that the tendencies of the biomechanical load were consistent with those of sensory evaluation of comfort. Finally, the biomechanical loads were calculated for various bathtub conditions and evaluated. It was found that the contributions of joint torques at the hip and neck to the comfort were dominant. It was also found that hip joint torques not only in the flexion/extension direction but also in the abduction/adduction direction were affected by the difference in bathtub length. It was suggested that the biomechanical load in the convex foot wall was smaller than those in the flat and concave ones for male bathers.
\end{abstract}

Keywords : Bathtub, Soaking posture, Biomechanical load, Joint torque, Biomechanics, Ergonomics

\section{Introduction}

Soaking in bathwater is an especially familiar custom in Japan. Its objectives are not only to warm the body but also to relieve fatigue and to get relaxed. For those objectives, comfort is required for soaking in bathwater. Several studies investigated comfort during soaking in bathwater. From the physiological viewpoint, changes in heart rate (Yoshimura et al., 1995; Oyake et al., 1999) and brain waves (Yanagihashi et al., 1996) during soaking in bathwater were investigated according to the changes in temperature, soaking time and method. From the biomechanical viewpoint, Kato et al. (2016) investigated how the soaking posture affects the body and mind from the perspective of biomechanics and neuroscience. They showed that joint torques and cerebral blood changed due to change in the posture. Since their results suggested that the reduction in joint torques might induce cerebral relaxation, it is important to investigate the biomechanical characteristics such as joint torques during soaking in bathwater in order to realize comfort. In addition, the biomechanical characteristics are considered to change based on the bathtub shape, since the soaking posture changes due to the bathtub shape. Therefore, in order to realize comfort during soaking in bathwater, the relationship between the biomechanical characteristics and the bathtub shape has to be clarified. If this relationship is fully clarified, it will be possible to improve bathtub shape design which will provide better comfort for the bather. From this viewpoint, Nakamura et al. (2018) proposed a biomechanical model in which a bather's body was represented as a link of body segments. In the model, the 
torque due to buoyancy and passive elastic joint moment were fully considered as well. Using basically the same model, Fujii et al. (2017) developed an algorithm to estimate reaction forces that act on a bather from a bathtub so that the biomechanical evaluation can be efficiently conducted without experiments in water. In addition, Nakamura et al. (2019) developed an algorithm to estimate the soaking posture itself as well as center of pressure of the reaction forces from the bathtub wall.

All above-mentioned biomechanical studies employed two-dimensional models in the sagittal plane of a bather. However, in actual soaking, a bather often takes three-dimensional postures, such as opening his/her legs, for which the two-dimensional sagittal plane model may not be sufficient. For more detailed investigation, it is necessary to take threedimensional postures during soaking into account.

The objectives of this study were to take three-dimensional postures during soaking in bathwater into account and to evaluate the biomechanical loads for three-dimensional soaking postures. In this paper, a three-dimensional biomechanical model and an experimental setup are described. Next, the biomechanical load is formulated using the model. Finally, the biomechanical loads and their components are evaluated in detail.

\section{Methods}

\subsection{Three-dimensional biomechanical model}

In order to calculate the biomechanical load during soaking in bathwater, a three-dimensional biomechanical model was considered in the present study. The definitions of force vectors acting on a bather and displacement vectors from the joint are shown in Fig. 1(a). In the model, a bather takes a soaking posture contacting the bathtub at the feet, hip, back and head. For simplicity, it was assumed that the upper limbs do not contact the bathtub. The joints at the ankle, knee, hip, lumbar (waist) and neck were considered. The joint torque components due to the reaction forces from the bathtub could be calculated as the outer products of the displacement vectors and reaction force vectors. For the calculation of buoyancy and gravitational forces, the swimming human simulation model SWUM (Nakashima et al., 2007) was employed, as shown in Fig 1(b). The soaking posture was assumed to be symmetrical. Therefore, all the joints except for the lower limb joints were assumed to have angles only in the flexion/extension in the sagittal plane. With respect to the lower limb joints, the hip and ankle joints had three degrees-of-freedom of the flexion/extension, abduction/adduction and external/internal rotations. The knee joint had flexion/extension only.

The flow to calculate muscular joint torques are shown in Fig. 2. At the start, experimental data are obtained by the experiment described later. The obtained experimental data are put into the model, and the joint torque components by gravity and buoyancy as well as reactions forces are calculated. In addition, from the calculated joint angles, passive elastic joint torques are calculated. The muscular joint torques, which were defined as the joint torques actively produced by the bather's muscles, could be calculated as the torques against the sums of torques by gravity and buoyancy, reactions forces and passive elastic joint torques.

\subsection{Calculation of passive elastic joint torques}

During soaking in bathwater, the muscular joint torques were generally considered to be small. In such situations, the passive elastic joint torques were considered to play an important role. Therefore, it was necessary to consider it

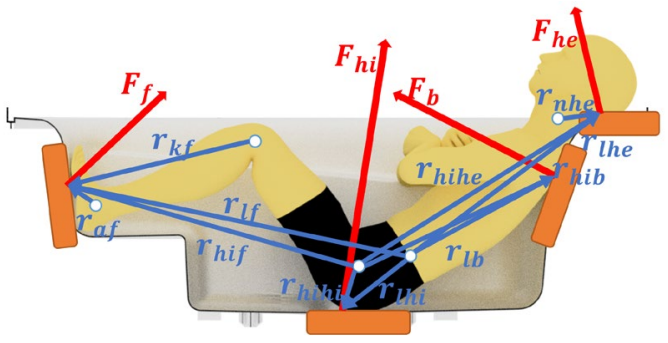

(a)

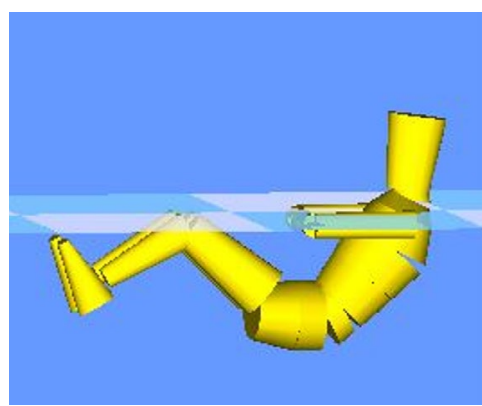

(b)

Fig. 1 Three-dimensional biomechanical model in the present study. (a) Definitions of reaction force vectors acting on the bather and displacement vectors from the joint, (b) image figure of the swimming human simulation model SWUM to calculate buoyancy. 
Nakashima, Fukuda, Fujii, Suzuki, Azuma and Nakamura,

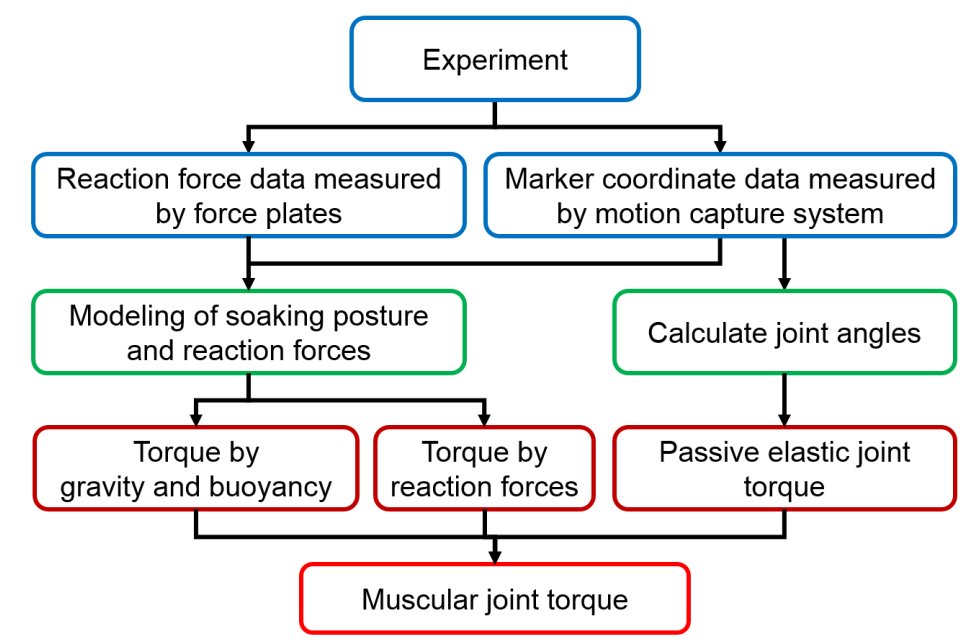

Fig. 2 Flow to calculate muscular joint torques. The muscular joint torques, which were defined as the joint torques actively produced by the bather's muscles, could be calculated as the torques against the sums of torques by gravity and buoyancy, reactions forces and passive elastic joint torques.

carefully in the present study. For the flexion/extension of the lumbar joint,

$$
T_{\text {Passive_Lumbar_fe }}[\mathrm{Nm}]=-0.5593 \exp \left(-3.8053 \theta_{L}\right)
$$

was used based on the experimental results by Beach et al. (2005). The symbol $\theta_{L}$ is the flexion/extension angle of the lumbar joint. For the flexion/extension of the neck joint,

$$
T_{\text {Passive_Neck_fe }}[\mathrm{Nm}]=\exp \left(2.1 \theta_{N}\right)-\exp \left(-2.3 \theta_{N}\right)
$$

was used based on experimental results by McGill et al. (1994). The symbol $\theta_{N}$ is the flexion/extension angle of the neck joint. For the flexion/extension of the hip, knee and ankle joints,

$$
\begin{aligned}
& T_{\text {Passive_Hip_fe }}[\mathrm{Nm}] \\
& \quad=\exp \left(1.4655-0.0034 \theta_{K}-0.0750 \theta_{H}\right) \\
& \quad-\exp \left(1.3403-0.0226 \theta_{K}+0.0305 \theta_{H}\right)+8.072-3.8 \\
& \begin{aligned}
T_{\text {Passive_Knee_fe }[} & \mathrm{Nm}] \\
& =\exp \left(1.800-0.0460 \theta_{A}-0.0352 \theta_{K}+0.0217 \theta_{H}\right) \\
& -\exp \left(-3.971-0.0004 \theta_{A}+0.0495 \theta_{K}-0.0128 \theta_{H}\right)-4.820 \\
& +\exp \left(2.220-0.150 \theta_{K}\right)+3.5 \\
T_{\text {Passive_Ankle_fe }}[\mathrm{Nm}] & \\
& =\exp \left(2.1016-0.0843 \theta_{A}-0.0173 \theta_{K}\right) \\
& -\exp \left(-7.9763+0.1949 \theta_{A}+0.0008 \theta_{K}\right)-1.762+1.2
\end{aligned}
\end{aligned}
$$

were used. The symbols $\theta_{H}, \theta_{K}$ and $\theta_{A}$ are the flexion/extension angles of the hip, knee and ankle joints, respectively. These formulas were derived by Fujii et al. (2017), in which the formulas by Riener and Edrich (1999) were modified with respect to the offset values based on the joint angles in the non-gravity position (Mount et al., 2003). In these formulas, not only the target joint angle itself, but also the effects of other joint angles were considered.

For the external/internal rotation and abduction/adduction of the hip joint as well as the abduction/adduction of the ankle joint,

$$
T_{\text {passive_other }}[\mathrm{Nm}]=\exp \left(k_{1}\left(\left(\theta-k_{5}\right)-k_{2}\right)\right)-\exp \left(k_{3}\left(k_{4}-\left(\theta-k_{5}\right)\right)\right)
$$


was used. The symbol $\theta$ is the target joint angle. This formula was based on the formula proposed by Yamazaki and Tanaka (2006). The values of the coefficients $k_{1}, k_{2}, k_{3}$ and $k_{4}$ are shown in Table 1 . These values were taken from the original formula by Yamazaki and Tanaka (2006). The coefficient $k_{5}$ in Eq. (6), on the other hand, was originally added in the present study. This coefficient corresponds to the horizontal offset of Eq. (6), and this offset value was expected to change according to the bather, especially based on sex. Therefore, this offset value was identified for each joint angle of each participant in the experiment described later.

It should be noted that the experimental results obtained in various different experiments were used together for the analysis of this study. Indeed, the experimental conditions, such as participants' age, sex, figures and so on, were not consistent in these experiments. This fact would become a limitation of this study. In order to solve this limitation, a comprehensive experiment to measure all the passive elastic joint torques for controlled participants will be necessary.

\subsection{Experimental setup}

A three-dimensional motion analysis system (Venus3D, Nobby Tech, Japan) and waterproofed three-dimensional force plates (TF-3040-W, Tech Gihan, Japan, 9253B11, Kistler, Switzerland) were used to measure the soaking postures and reaction forces from a bathtub on the feet, hip, trunk and neck, as shown in Fig. 3(a). Fifteen self-luminous markers were put on the left side of the participants' bodies at the top of head, shoulder acromion, axilla, sternum lower end, waist, iliac crest, great trochanter, femur, lateral side of knee, tibia, lateral malleolus, foot, fifth metatarsal, abdomen and ear. Data were obtained for $30 \mathrm{~s}$ at $30 \mathrm{~Hz}$ and low-pass filtered at $10 \mathrm{~Hz}$ using a fourth-order Butterworth filter. Then data were averaged with recorded time.

The directions of body segments in the biomechanical model were calculated from the three-dimensional marker coordinate data. The body segments of the trunk including the neck were placed in the sagittal plane. The body segments of the left lower limb were placed in the three-dimensional space. The body segments of the right lower limb were also placed in the three-dimensional space by flipping the left ones horizontally, based on the assumption of symmetry. The joint angles of the lower limb joints were calculated using the Joint Coordinate System (Robertson et al., 2014). In the actual calculation, the marker coordinate system was transformed into the anatomical coordinate system (Winter, 2009). Since this transformation matrix does not depend on the posture, a reference posture to calculate the matrix was measured on land with several additional markers on the participants' body before the soaking experiment.

Table 1 Coefficients for the passive elastic joint torque of Eq. (6). These values were taken from the original formula by Yamazaki and Tanaka (2006).

\begin{tabular}{ccccc}
\hline Joint angle & $k_{1}$ & $k_{2}$ & $k_{3}$ & $k_{4}$ \\
\hline Hip external/internal rotation & 2.6 & -0.1 & 4.6 & 0.1 \\
Hip abduction/adduction & 0.0 & 0.0 & 3.9 & 0.0 \\
Ankle abduction/adduction & 2.3 & 0.0 & 1.8 & -0.1 \\
\hline
\end{tabular}

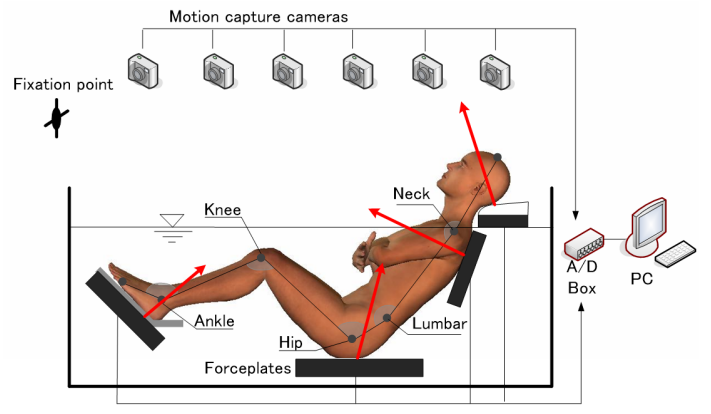

(a)

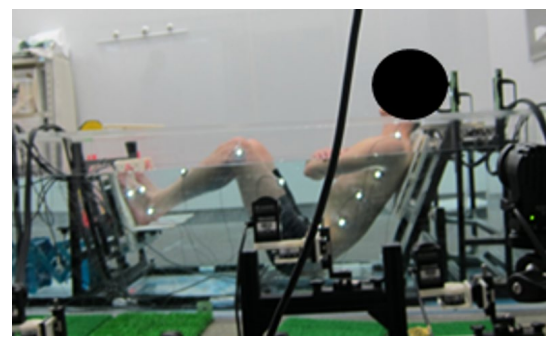

(b)

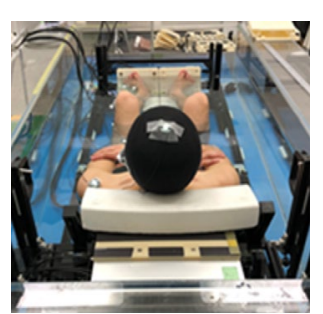

(c)

Fig. 3 Experimental setup. (a) Schematic view, (b) photograph from the side, (c) photograph from the top. Fifteen selfluminous markers were put on the left side of the participants' bodies at the top of head, shoulder acromion, axilla, sternum lower end, waist, iliac crest, great trochanter, femur, lateral side of knee, tibia, lateral malleolus, foot, fifth metatarsal, abdomen and ear. 


\subsection{Experimental conditions}

Eight healthy adults (four males and four females) participated in the present study. All the participants gave their written informed consent. The study was approved by the TOTO Ltd. ethics board (No. 08_20181001, Study of biomechanical evaluation for sex difference in bathing postures). With respect the bathtub length, two conditions (L: long and S: short) were prepared for each participant. Since the heights of the participants were widely different (1.52 $\mathrm{m} \sim 1.80$ $\mathrm{m})$, the nondimensional bathtub lengths normalized by the participants' heights were calculated as shown in Table 2 , and each actual bathtub length was selected so that each nondimensional bathtub length becomes as constant as possible. As a result, the nondimensional bathtub lengths became $0.64 \sim 0.72$ for L (long) and $0.53 \sim 0.59$ for $\mathrm{S}$ (short), respectively. In addition to the bathtub length, three shapes of bathtub foot wall were prepared, as shown in Fig. 4. For the convex shape, hip joints of a participant were expected to take more abducted and externally rotated position. For the concave shape, vice versa.

The participants were asked to take their most comfortable postures in the bathtub. They could freely determine their hip and feet positions in the bathtub. They were only asked to cross their arms to eliminate the effect of the bathtub on the upper limbs. Then they were asked to evaluate their comfort by the SD method, that is, on a scale of -3 (most uncomfortable) to 3 (most comfortable) for each condition. Note that the water volume was adjusted for each participant so that the level of water surface became at his/her chest.

In addition to the above main experiment, a supplementary experiment to measure the passive elastic joint torques was also conducted for the same participants. The experimental setup for the experiment is shown in Fig. 5. There was no bathtub foot wall in this experiment. The hip position on the bathtub for each participant was determined so that the knee flexion angles in this experiment became the same as those in the main experiment as possible. Since there was no bathtub foot wall, the participants were asked to take joint angles for the abduction/adduction and external/internal rotation at the hip and abduction/adduction at the ankle without muscle forces (completely without effort). In this condition, the three-dimensional body postures and reaction forces from the bathtub were measured the same as the main experiment.

Table 2 Participants and bathtub lengths in the experiment. Each value in the bracket represents nondimensional bathtub length normalized by participant's height.

\begin{tabular}{ccccccc}
\hline Participant No. & Gender & Age & Height & Weight & Bathtub length: L & Bathtub length: S \\
\hline 1 & Male & 30 & $1.66 \mathrm{~m}$ & $57 \mathrm{~kg}$ & $1150 \mathrm{~mm}(0.69)$ & $950 \mathrm{~mm}(0.57)$ \\
2 & Male & 28 & $1.68 \mathrm{~m}$ & $65 \mathrm{~kg}$ & $1150 \mathrm{~mm}(0.68)$ & $950 \mathrm{~mm}(0.57)$ \\
3 & Male & 25 & $1.70 \mathrm{~m}$ & $55 \mathrm{~kg}$ & $1150 \mathrm{~mm}(0.68)$ & $950 \mathrm{~mm}(0.56)$ \\
4 & Male & 27 & $1.80 \mathrm{~m}$ & $76 \mathrm{~kg}$ & $1150 \mathrm{~mm}(0.64)$ & $950 \mathrm{~mm}(0.53)$ \\
5 & Female & 25 & $1.52 \mathrm{~m}$ & $42 \mathrm{~kg}$ & $1100 \mathrm{~mm}(0.72)$ & $900 \mathrm{~mm}(0.59)$ \\
6 & Female & 26 & $1.54 \mathrm{~m}$ & $45 \mathrm{~kg}$ & $1100 \mathrm{~mm}(0.71)$ & $900 \mathrm{~mm}(0.58)$ \\
7 & Female & 28 & $1.62 \mathrm{~m}$ & $50 \mathrm{~kg}$ & $1150 \mathrm{~mm}(0.71)$ & $950 \mathrm{~mm}(0.59)$ \\
8 & Female & 25 & $1.65 \mathrm{~m}$ & $53 \mathrm{~kg}$ & $1150 \mathrm{~mm}(0.70)$ & $950 \mathrm{~mm}(0.58)$ \\
\hline
\end{tabular}

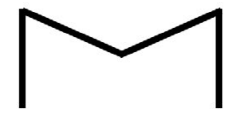

(top view)

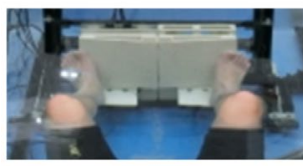

Convex

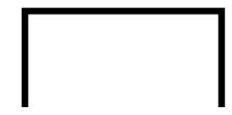

(top view)

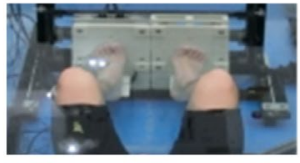

Flat

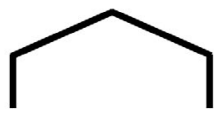

(top view)

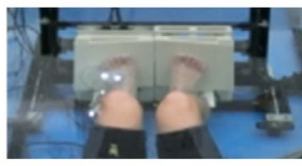

Concave

Fig. 4 Three shapes of bathtub foot wall. Convex, Flat and Concave. For the convex shape, hip joints of a participant were expected to take more abducted and externally rotated position. For the concave shape, vice versa. 


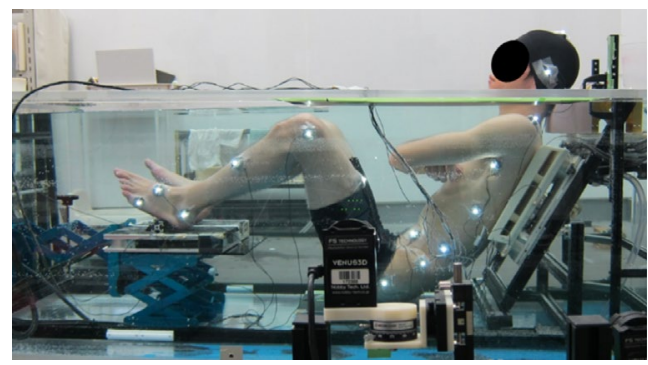

Fig. 5 Experimental setting for supplementary experiment to measure offset values of passive elastic joint torques. There was no bathtub foot wall in this experiment. The participants were asked to take joint angles for the abduction/adduction and external/internal rotation at the hip and abduction/adduction at the ankle without muscle forces (completely without effort).

\section{Results and Discussion}

\subsection{Postures, forces and sensory evaluation}

The experimental results of soaking postures and reaction forces from the bathtub wall for six combinations of the bathtub length ( $\mathrm{L}$ and $\mathrm{S}$ ) and foot wall shape (Convex, Flat and Concave) (Participant 1) are shown in Fig. 6. For each case, the top figure is the top view and the bottom is the side view. It was found that the adduction/abduction angle and internal/external rotation angle of the hip joint changed according to the foot wall shape. It was also found that the
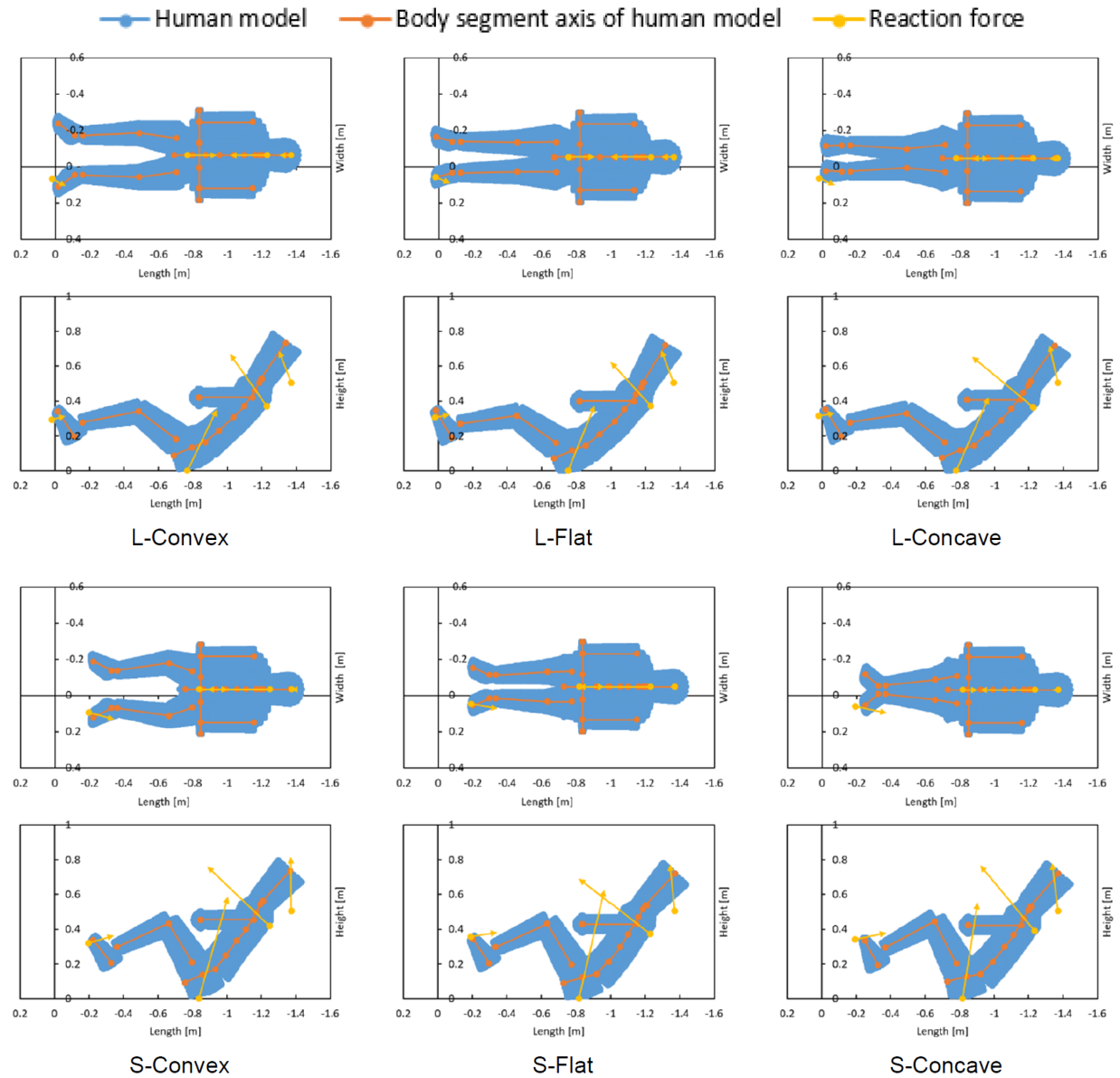

Fig. 6 Experimental results of soaking postures and reaction forces from the bathtub wall for six combinations of bathtub length (L and S) and foot wall shape (Convex, Flat and Concave) (Participant 1). For each case, the top figure is the top view and the bottom is the side view. It was found that the adduction/abduction angle and internal/external rotation angle of the hip joint changed according to the foot wall shape. It was also found that the flexion/extension angle of the knee and hip joints changed according to the bathtub length. 
flexion/extension angle of the knee and hip joints changed according to the bathtub length.

The results of sensory evaluation are shown in Fig. 7. For the male participants (the left figure), it was found that the longer bathtub was more preferred than the shorter one, and that the convex foot wall was most preferred, the flat was next and the concave was the worst. For the female participants, on the other hand, an apparent tendency could not be found.

\subsection{Offset of passive elastic joint torques}

By putting the data measured in the supplementary experiment to the biomechanical model, the joint torques of the participants were calculated. It was assumed that the muscular joint torques were zero for the abduction/adduction and external/internal rotation at the hip and the abduction/adduction at the ankle. Therefore, the offset values $\left(k_{5}\right.$ in Eq. (6)) were determined so that the calculated muscular joint torques became zero. The results are shown in Table 3 . It was found that negative values could be seen in many cases for male participants, while positive values in many cases for female participants. It means that the more abducted and externally rotated angles at the hip and more abducted angles at the ankle were natural positions for the male participants. These determined values were used for the calculation of the biomechanical load in the next section.

\subsection{Biomechanical load}

The biomechanical load as a comprehensive and quantitative indicator of comfort was defined in this study. First, it was assumed that the biomechanical load was represented by the sum of muscular joint torques and passive elastic joint torques since these joint torques were perceived by a bather as undesirable physical tension. This biomechanical load will be low when a bather feels comfort. The biomechanical load $T_{B L}$ is represented by the following equation:
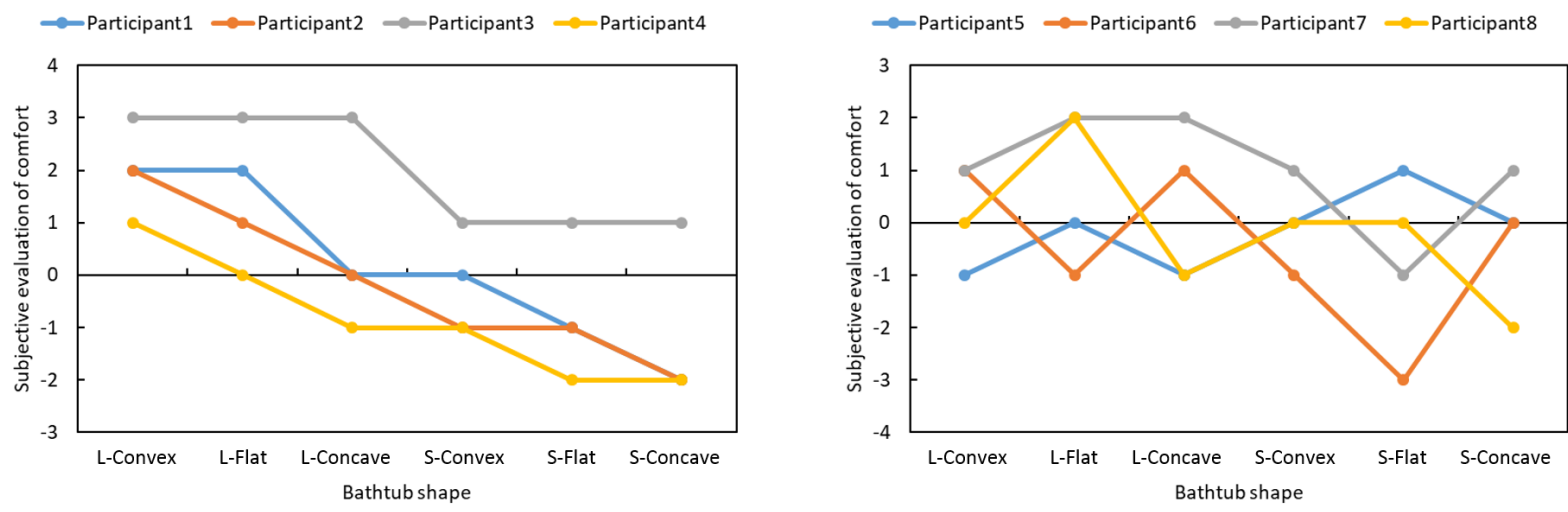

Fig. 7 Results of sensory evaluation. The results of male participants are shown in the left, while those of female participants are shown in the right.

Table 3 Experimental results of offset values of passive elastic joint torques. The numbers in red are negative values. It was found that negative values could be seen in many cases for male participants, while positive values in many cases for female participants.

\begin{tabular}{ccccccccccc}
\hline \multirow{2}{*}{ Joint } & \multirow{2}{*}{ Direction } & Bathtub & \multicolumn{1}{c}{ Marticipant No. } \\
& & length & 1 & 2 & 3 & 4 & 5 & 6 & 7 & 8 \\
\hline \multirow{2}{*}{ Hip } & Internal & $\mathrm{L}$ & -6 & -8 & -16 & -7 & 13 & 31 & 2 & 2 \\
& \multirow{2}{*}{ rotation } & $\mathrm{S}$ & -4 & -2 & -8 & -2 & 7 & 7 & 10 & -5 \\
\hline \multirow{2}{*}{ Hip } & \multirow{2}{*}{ Adduction } & $\mathrm{L}$ & 1 & -59 & -29 & -8 & 13 & 17 & 2 & -7 \\
& & $\mathrm{~S}$ & -11 & -24 & -40 & -7 & 13 & 32 & 10 & -1 \\
\hline \multirow{2}{*}{ Ankle Adduction } & $\mathrm{L}$ & 3 & -10 & -3 & 0 & 4 & -18 & 0 & 2 \\
& $\mathrm{~S}$ & -4 & -22 & -16 & 3 & 21 & -26 & 8 & -1 \\
\hline
\end{tabular}




$$
T_{B L}=\sum_{i=1}^{8}\left(W_{\text {muscular_ } i} \cdot\left|T_{\text {muscular_ } i}\right|\right)+\sum_{i=1}^{8}\left(W_{\text {passive } \_i} \cdot\left|T_{\text {passive__ }}\right|\right)
$$

The symbols $T_{\text {muscular } i}$ and $T_{\text {passive } i}$ are the $i$-th muscular joint torque and passive elastic joint torque, respectively. The following eight joint angles $(i=1 \sim 8)$ were considered: flexion/extension at the neck, lumbar, hip, knee and ankle, abduction/adduction at the hip and ankle, and hip external/internal rotation. The symbols $W_{\text {muscular } i}$ and $W_{\text {passive_i }}$ are the weighting coefficients for the joint torques. In order to determine these weighting coefficients, the experimental results of sensory evaluation were used. The weighting coefficients were determined so that the magnitude relationship of the sensory evaluation was inversely consistent with that of the biomechanical load. For example, Participant 1's comfort scores were L-Convex $=$ L-Flat $>$ L-Concave $=$ S-Convex $>$ S-Flat $>$ S-Concave, as shown in Fig. 7. For this case, the weighting coefficients should be determined so that the magnitude relationship of the biomechanical load becomes L-Convex $\approx$ L-Flat $<$ L-Concave $\approx$ S-Convex $<$ S-Convex $<$ S-Flat $<$ S-Concave. In addition, the weighting coefficients were assumed to be basically constant for all the participants to maintain generality. Therefore, the weighting coefficients reasonable for all the participants were investigated by comparing the magnitudes of the 16 joint torques with the results of sensory evaluation. As a result, a set of weighting coefficients, by which magnitude relationships of the sensory evaluation and the biomechanical load were completely consistent with each other for five (Participants 2, 3, 4, 6 and 7) among eight participants, was found. The results are shown in Table 4 as numbers in black. For those five participants, the biomechanical load was calculated with these values of weighting coefficients. For other two participants (Participants 1 and 8), different sets of weighting coefficients, by which magnitude relationships of the sensory evaluation and the biomechanical load were completely consistent with each other, were found respectively, by slightly modifying the values of weighting coefficients. Specifically, the modified values for Participant 1 are shown in red in Table 4, while the modified values for Participant 8 are shown in blue. These weighting coefficients were used to calculate the biomechanical load for these two participants. For Participant 5 , however, it was not possible to find such weighting coefficients. This is probably because this participant' height was smallest. Indeed the other participants felt comfort with the longer (L) bathtub, while Participant 5 felt comfortable with the shorter (S) bathtub.

The biomechanical loads for all the participants except for Participant 5 were calculated, and contributions of joint torques to the biomechanical load were investigated. For this purpose, the magnitude of each weighted joint torque for each participant was calculated and averaged for all the bathtub conditions. Then the magnitudes of all the joint torques were ranked. This ranking was conducted for all the participants and then the ranks were averaged. It represents the contributions of the weighted joint torques to the biomechanical load. In other words, it represents the order of joint torques with which the participants were (unconsciously) concerned during soaking in bathwater. The results are shown in Table 5. It was found that the contributions of joint torques at the hip and neck were dominant. The possible reason for the hip joints is that the hip joints have three degrees-of-freedom and therefore the joint angles are affected by the change both in the bathtub length and in the foot wall shape. The possible reason for the neck joint is that bathers would like to reduce the burden of the muscles around the neck, which tend to be tired from supporting the head.

The effects of bathtub length on the biomechanical load are shown in Fig. 8. In this figure, the joint torques which

Table 4 Determined weighting coefficients for muscular joint torques and passive elastic joint torques. The numbers in black are common values for five (Participants 2,3,4,6 and 7) among eight participants. The numbers in red are for Participant 1, while those in blue are for Participant 8.

\begin{tabular}{lcc}
\hline & Muscular joint torque & Passive elastic joint torque \\
\hline Hip flexion/extension & 4 & $0(7)$ \\
Knee flexion/extension & 0 & $0(88)$ \\
Ankle flexion/extension & $7(14)$ & $0(300)$ \\
Lumbar flexion/extension & $1(2)$ & $0(2)$ \\
Neck flexion/extension & $44(0,88)$ & $69(46,0)$ \\
Hip external/internal rotation & $5(20)$ & 0 \\
Hip abduction/adduction & $14(21)$ & $34(0)$ \\
Ankle abduction/adduction & $22(0,0)$ & $21(42)$ \\
\hline
\end{tabular}


Nakashima, Fukuda, Fujii, Suzuki, Azuma and Nakamura,

Journal of Biomechanical Science and Engineering, Vol.15, No.4 (2020)

Table 5 Ranking of weighted joint torques with respect to the contribution to the biomechanical load. It represents the contributions of the weighted joint torques to the biomechanical load. In other words, it represents the order of joint torques with which the participants were (unconsciously) concerned during soaking in bathwater.

\begin{tabular}{ccc}
\hline Rank & Joint motion & Torque type \\
\hline 1st & Hip abduction/adduction & Muscular joint torque \\
2nd & Hip flexion/extension & Muscular joint torque \\
3rd & Neck flexion/extension & Muscular joint torque \\
4th & Hip abduction/adduction & Passive elestic joint torque \\
5th & Neck flexion/extension & Passive elestic joint torque \\
\hline
\end{tabular}

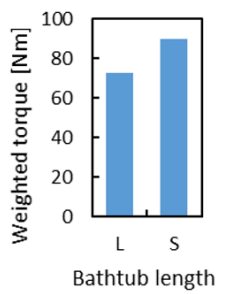

(a)

Hip abduction/adduction Muscular joint torque (1st place)

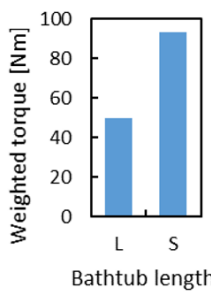

(b)

Hip flexion/extension Muscular joint torque (2nd place)

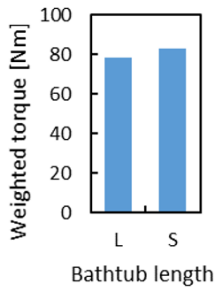

(c)

Neck flexion/extension Muscular joint torque (3rd place)

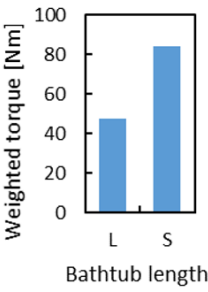

(d)

Hip abduction/adduction Passive elastic joint torque (4th place)

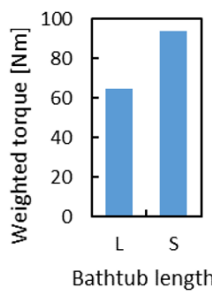

(e)

Neck flexion/extension Passive elastic joint torque (5th place)

Fig. 8 Effects of bathtub length on the biomechanical load. Five joint torques which contributed most to the biomechanical load are shown for the long (L) and short (S) bathtub conditions. It was found that the biomechanical load in the long bathtub was smaller than that in the short one for all the joint torques.

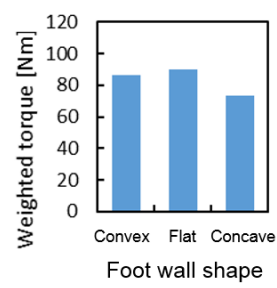

(a)

Hip abduction/adduction Muscular joint torque (1st place)

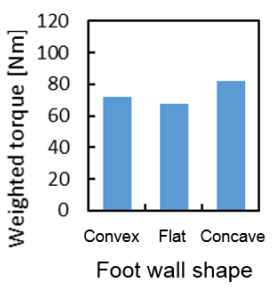

(b)

Hip flexion/extension Muscular joint torque (2nd place)

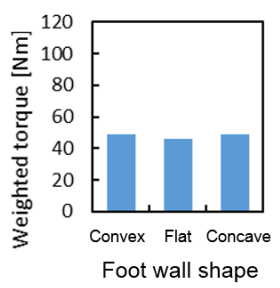

(c)

Neck flexion/extension Muscular joint torque (3rd place)

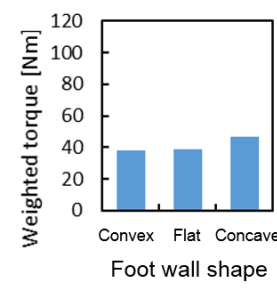

(d)

Hip abduction/adduction Passive elastic joint torque (4th place)

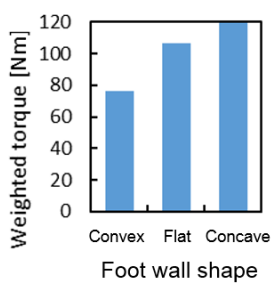

(e)

Neck flexion/extension Passive elastic joint torque (5th place)

Fig. 9 Effects of bathtub foot wall shape on the biomechanical load for the male participants. Five joint torques which contributed to the biomechanical load are shown for the three (Convex, Flat and Concave) foot wall conditions.

were ranked high in Table 5 are shown for the long (L) and short (S) bathtub conditions. The values were averaged for all the participants. It was found that the joint torques in the long bathtub condition were smaller than those in the short bathtub conditions for all the joint torques. Interestingly, it was also found that hip joint torques not only in the flexion/extension direction but also in the abduction/adduction direction were affected by the difference in the bathtub length. This is because the hip abduction angle changes as well according to the change in the bathtub length.

The effects of bathtub foot wall shape on the biomechanical load for the male participants are shown in Fig. 9. In this figure, the joint torques which were ranked high in Table 5 are shown for the three (Convex, Flat and Concave) foot wall conditions. The values were averaged for all the male participants. Although a clear tendency could not be seen, the joint torques in the concave foot wall were larger than those in the flat one for all the joint torques except for (a), and the joint torques in the convex foot wall were smaller than those in the flat foot wall for the three joint torques ((a), (d) and 
(e)). It suggests that the male bathers preferred the flat foot wall more than the concave one, and preferred the convex foot wall more than the flat one. For the female participants, on the other hand, any general tendency could not be seen. It suggests that there is a difference between male and female in the effects of bathtub foot wall shape on the joint torques. This suggestion raises a problem how to realize a bathtub comfortable both for male and female, although a bathtub in a house is generally used both by male and female. This problem might be solved, for example, by producing a step at the foot wall to provide two different foot wall shapes according to the body height (lower step for female and higher step for male).

The limitation of this study is that the insufficient number of participants. The range of age for the participants were particularly narrow ( 25 to 30 years old). If more data for more participants can be obtained, more general tendency as well as variation due to individual differences in weighting coefficients of the biomechanical load may be found. In addition, it will also be a future task to investigate the relationship between the weighting coefficients in the biomechanical load and physical properties of participants, such as segment lengths, weights, and characteristics of the passive elastic joint moments.

\section{Conclusions}

In the present study, the joint torques during soaking in bathwater were calculated in consideration of threedimensional postures of the bather. In addition, the biomechanical load during soaking was defined as the sum of weighted joint torques, and the weighting coefficients were determined so that the tendencies of the biomechanical load were consistent with those of sensory evaluation of comfort. Finally, the biomechanical loads were calculated for various bathtub conditions and evaluated. It was found that the contributions of joint torques at the hip and neck to the comfort were dominant. It was also found that hip joint torques not only in the flexion/extension direction but also in the abduction/adduction direction were affected by the difference in the bathtub length. It was suggested that the biomechanical load in the convex foot wall was smaller than those in the flat and concave ones for male bathers.

\section{References}

Beach, T. A. C., Parkinson, R. J., Stothart, J. P. and Callaghan, J. P., Effects of prolonged sitting on the passive flexion stiffness of the in vivo lumbar spine, The Spine Journal, Vol.5, No.2 (2005), pp. 145-154.

Fujii, T., Kamada, A., Nakamura, R., Kato, T. and Nakashima, M., Development of an algorithm to estimate reaction forces from bathtub during soaking in the bathwater, Mechanical Engineering Journal, Vol.4, No.5 (2017), DOI: 10.1299/mej.17-00243

Kato, T., Sato, M., Matsushita, H., Nozawa, T. and Kawashima, R., Effects of sitting posture in bathtub bathing on joint torque and brain activities, Journal of Japanese Society for Medical and Biological Engineering (in Japanese), Vol.54, No.1 (2016), pp22-27.

McGill, S. M., Jones, K., Bennett, G. and Bishop P. J., "Passive stiffness of the human neck in flexion, extension, and lateral bending”, Clinical Biomechanics, Vol.9, No.3 (1994), pp.193-198.

Mount, F. E., Whitmore, M. and Stealey, S. L., Evaluation of Neutral Body Posture on Shuttle Mission STS-57 (SPACEHAB-1), NASA Technical Memorandum 104805 (2003).

Nakamura, R., Kato, T., Sato, M., Fujii, T. and Nakashima, M., Evaluation of soaking postures in bathwater using a biomechanical model considering buoyancy and passive elastic joint moment, Mechanical Engineering journal, Vol.5, No.3 (2018), DOI:10.1299/mej.18-00006

Nakamura, R., Yoneda, T., Kato, T. and Nakashima, M., Development of an algorithm to estimate soaking posture in the bathwater, Mechanical Engineering Journal, Vol.6, No.4 (2019), DOI: 10.1299/mej.18-00532

Nakashima, M., Miura, Y. and Satou, K., Development of swimming human simulation model considering rigid body dynamics and unsteady fluid force for whole body, Journal of Fluid Science and Technology, Vol.2 (2007), DOI: $10.1299 /$ jfst. 2.56

Oyake, Y., Otsuka, S., Yamaguchi, I., Sugishita, Y., Matsuzaki, J., Kato, S. and Ishimoto, A., Change of the autonomic nerve activity by the bathing. The examination by the heartbeat variability analysis, Therapeutic Research, Vol.20, No.2 (1999), pp.441-443 (in Japanese).

Riener, R. and Edrich, T., Identification of passive elastic joint moments in the lower extremities, Journal of 
Nakashima, Fukuda, Fujii, Suzuki, Azuma and Nakamura, Journal of Biomechanical Science and Engineering, Vol.15, No.4 (2020)

Biomechanics, Vol.32, No.5 (1999), pp.539-544.

Robertson, G. E., Caldwell, G. E., Hamill, J., Kamen, G. and Whittlesey, S. N., Research Methods in Biomechanics Second Edition (2014), pp.35-59, Human Kinetics.

Winter, D. A., Biomechanics and Motor Control of Human Movement Fourth Edition (2009), pp.180-187, John Wiley \& Sons.

Yamazaki, N. and Tanaka, T., Development of a human joint imitated dummy, Biomechanisms, Vol.18 (2006), pp.175185 (in Japanese).

Yanagihashi, R., Fujiwara, T. and Ide, K., Physiological and psychological effects of hot bath: Fluctuation analysis of alpha wave of EEG, Journal of Japan Society of Nursing Research (in Japanese), Vol.19, No.3 (1996), pp7-12.

Yoshimura, T., Tamura, T., Osa, A., Nakajima, K., Miike, H. and Togawa, T., Heart rate variability during bathing andcomfort of bathing, Reports of the Institute for Medical\& Dental Engineering, Vol.29 (1995), pp.95-103. 